\title{
Transcription of hexose transporters of Saccharomyces cerevisiae is affected by change in oxygen provision
}

\author{
Eija Rintala*, Marilyn G Wiebe, Anu Tamminen, Laura Ruohonen and \\ Merja Penttilä
}

Address: VTT, Technical Research Centre of Finland, P.O. Box 1000, FI-02044 VTT, Finland

Email: Eija Rintala* - eija.rintala@vtt.fi; Marilyn G Wiebe - marilyn.wiebe@vtt.fi; Anu Tamminen - anu.tamminen@vtt.fi; Laura Ruohonen - laura.ruohonen@vtt.fi; Merja Penttilä - merja.penttila@vtt.fi

* Corresponding author

Published: 28 March 2008

BMC Microbiology 2008, 8:53 doi:10.1186/|47|-2/80-8-53
Received: 16 October 2007

Accepted: 28 March 2008

This article is available from: http://www.biomedcentral.com/I47I-2/80/8/53

(C) 2008 Rintala et al; licensee BioMed Central Ltd.

This is an Open Access article distributed under the terms of the Creative Commons Attribution License (http://creativecommons.org/licenses/by/2.0), which permits unrestricted use, distribution, and reproduction in any medium, provided the original work is properly cited.

\begin{abstract}
Background: The gene family of hexose transporters in Saccharomyces cerevisiae consists of 20 members; 18 genes encoding transporters (HXTI-HXTI 7, GAL2) and two genes encoding sensors (SNF3, RGT2). The effect of oxygen provision on the expression of these genes was studied in glucose-limited chemostat cultivations $\left(\mathrm{D}=0.10 \mathrm{~h}^{-1}, \mathrm{pH} 5,30^{\circ} \mathrm{C}\right)$. Transcript levels were measured from cells grown in five steady state oxygen levels $\left(0,0.5, \mathrm{I}, 2.8\right.$ and $\left.20.9 \% \mathrm{O}_{2}\right)$, and from cells under conditions in which oxygen was introduced to anaerobic cultures or removed from cultures receiving oxygen.
\end{abstract}

Results: The expression pattern of the HXT gene family was distinct in cells grown under aerobic, hypoxic and anaerobic conditions. The transcription of HXT2, HXT4 and HXT5 was low when the oxygen concentration in the cultures was low, both under steady state and non-steady state conditions, whereas the expression of HXT6, HXT/3 and HXT/5//6 was higher in hypoxic than in fully aerobic or anaerobic conditions. None of the HXT genes showed higher transcript levels in strictly anaerobic conditions. Expression of HXT9, HXTI4 and GAL2 was not detected under the culture conditions studied.

Conclusion: When oxygen becomes limiting in a glucose-limited chemostat cultivation, the glucose uptake rate per cell increases. However, the expression of none of the hexose transporter encoding genes was increased in anaerobic conditions. It thus seems that the decrease in the moderately low affinity uptake and consequently the relative increase of high affinity uptake may itself allow the higher specific glucose consumption rate to occur in anaerobic compared to aerobic conditions.

\section{Background}

The hexose transporter gene family in Saccharomyces cerevisiae contains the sugar transporter genes HXT1 to HXT17, GAL2 and the glucose sensor genes SNF3 and RGT2 $[1,2]$. The proteins encoded by HXT1 to HXT4 and
HXT6 to HXT7 are considered to be the major hexose transporters in S. cerevisiae. The MC996A yeast strain lacking these six transporters and the protein encoded by HXT5 (referred to as a hxt null mutant) is unable to grow on glucose [3]. Based on their $K_{m}$ values and their ability 
to restore growth on glucose when the respective genes are expressed individually in the hxt null mutant strain, these transporters have been classified as high (Hxt6p, Hxt7p), moderately low (Hxt2p, Hxt4p) and low (Hxt1p, Hxt3p) affinity transporters. However, Hxt2p in cells grown on low glucose concentrations exhibits both high and low affinity transport kinetics [4]. Gal2p is able to transport glucose with high affinity but the gene encoding it is expressed only when galactose is present [4,5]. In the CEN.PK2-1C strain, deletion of only HXT1 to HXT7 is not enough to completely abolish growth on glucose, fructose or mannose [6]. Only deletion of all seventeen of the HXT genes and GAL2 produces a strain unable to grow on these sugars, and overexpression of any individual gene encoding a hexose transporter, except HXT12 in this multiple deletant restores growth on at least one of the hexoses: glucose, fructose, mannose or galactose [6].

The major glucose transporters are regulated at transcriptional level by the extracellular glucose concentration [7]. The transcription factor Rgt 1 p represses the genes encoding these transporters in the absence of glucose, but it is also required for full induction of HXT1 on high levels of glucose [8]. Paralogous proteins Mth1p and Std1p are necessary for Rgt1p to act as a repressor. Release of the repression requires removal of Mth1p and Std1p as well as phosphorylation of Rgt1. The glucose signal mediated by the Snf3p and Rgt2p sensors stimulates the degradation of Mth1p and Std1p, while the glucose signal mediated by the G-protein-coupled receptor Gpr1p leads to activation of protein kinase A, which phosphorylates Rgt1 and releases the repression of HXT genes [9-15]. In addition, high affinity transporters are repressed in high levels of glucose via the Snf1p-Mig1p glucose repression pathway. Mig1p binds directly to the promoters of HXT2 and HXT4 and also represses the expression of MTH1 and SNF3 $[15,16]$.

Expression of HXT5 is regulated by growth rate rather than the external glucose concentration $[17,18]$. It is expressed upon decrease in the growth rate of cells in glucose batch cultivations, at low dilution rates in glucose-limited chemostat cultivations, on non-fermentable carbon sources and during sporulation $[17,19]$. The regulation is mediated by STRE and HAP elements in the promoter region of HXT5. The STRE elements are needed for induction at low growth rates and during growth on ethanol, and the HAP elements for growth on ethanol or glycerol [17]. The Hxt5p transporter shows moderately low affinity for glucose [19].

The hexose transporters encoded by HXT8 to HXT17 have not been studied to the same extent as the major HXT genes. Many of the functions assigned to these transporters do not directly relate to sugar utilisation. Diderich and co-workers [20] were not able to detect the expression of these genes when probing total RNA from glucose-limited chemostat cultivations. It is known, however, that the regulation of all but HXT11 and HXT12 is controlled by glucose [7]. The transporters encoded by HXT9 and HXT11 are also shown to be involved in pleiotropic drug resistance [21], and the promoter of HXT17 is a target for Mac1p transcription factor, which regulates high-affinity copper uptake genes under copper-deficient conditions $[22,23]$. Greatrix and co-workers [24] demonstrated that HXT5, HXT13 and HXT15 are induced in the presence of non-fermentable carbon sources, and that HXT17 is upregulated when yeast cells are grown on medium containing raffinose and galactose at $\mathrm{pH} 7.7$ but not at $\mathrm{pH}$ 4.7. In addition, Hxt9p and Hxt10p are able to transport arsenic trioxide into the cell, as do the major hexose transporters [25].

In addition to regulation at transcriptional level, inactivation of Hxt proteins takes place under certain conditions such as starvation or in the presence of high concentrations of glucose [7]. Degradation via endocytosis, autophaghy, and transport to the vacuole has been shown for Hxt2p, Hxt5p, Hxt6p and Hxt7p [26-30].

In glucose-limited chemostats, the specific glucose consumption rate is inversely related to the oxygen provided to the system [31,32]. Under oxygen-limited or anaerobic conditions, energy is provided by respiro-fermentative and fermentative metabolism, respectively. The biomass yield on glucose is lower and the cells must transport more glucose per unit time to be able to grow at the same rate in anaerobic or oxygen-limited conditions as when the metabolism is fully respirative. It is not clear what modifications in the cell are responsible for the higher glucose uptake. In addition, in spite of numerous studies, the mechanism(s) controlling glycolysis in yeast is still unknown. Control of individual steps or enzymes of this pathway, such as the phosphorylation of glucose and the control of phosphofructokinase have been extensively studied [33-35]. However, it is likely that the control is distributed over a number of steps, since the overexpression of individual glycolytic enzymes, or of all, did not enhance glycolytic flux [36-38]. Glucose transport has been suggested to play a major role in the control of glycolysis [39], and shown to be highly important for the dynamics of glycolysis with substantial control over the frequency of glycolytic oscillations [38]. Additionally, glucose transport may control the flux of glycolysis, even at high external glucose concentrations if transport capacity is reduced, as is the case in a strain that expresses a chimera between Hxt1p and Hxt7p and no other hexose transporter $[40,41]$. 
Previously, we have assessed the role of oxygen in the physiology of $S$. cerevisiae by studying the metabolite and transcript levels related to central carbon metabolism [32]. In the present study we extend the analysis to glucose transport to determine if and how hexose transporters are affected when oxygen becomes limiting for growth. We probed cells from glucose-limited chemostat cultivations of CEN.PK113-1A at five different oxygen levels for the expression of HXT1 to HXT11, HXT13 to HXT17, GAL2, SNF3 and RGT2. In addition, we studied the expression of these genes under conditions in which oxygen was removed from cultivations receiving it or introduced to anaerobic cultivations.

\section{Results \\ Transcription of HXT genes in cells at steady state exposed to different oxygen levels}

Five different inflow gas oxygen levels (20.9, 2.8, 1.0, 0.5 and $0 \%$ ) were used to study the response in expression of genes encoding hexose transporters and glucose sensors to external oxygen in glucose-limited chemostat cultures of CEN.PK113-1A. The specific glucose consumption rates were 6.6, 8.0, 11.4, 14.3 and $37.1 \mathrm{Cmmol} \mathrm{g}$ biomass $^{-1} \mathrm{~h}^{-1}$ for chemostat cultures receiving 0.5, 1.0, 2.8 and 20.9\% oxygen, respectively [32]. Extracellular glucose was not detected. The strongest expression signals were obtained from HXT6, HXT7 and HXT10. The signals of HXT9, HXT14 and GAL2 transcripts were below the detection limit in these conditions.

Statistical differences $(\mathrm{p}<0.05)$ in the expression levels of all the hexose transporter encoding genes were detected between at least some of the oxygen concentrations studied (Figure 1). HXT2, HXT4 and HXT5 were significantly more highly expressed in aerobic conditions compared to hypoxic $\left(2.8,1.0\right.$ and $\left.0.5 \% \mathrm{O}_{2}\right)$ or anaerobic conditions. Each of these genes showed some increase in expression already at $2.8 \% \mathrm{O}_{2}$. HXT2 also had higher transcript levels in anaerobic conditions compared to the hypoxic conditions of 1.0 and $0.5 \% \mathrm{O}_{2}$. HXT1, HXT8, HXT11 and HXT17 had higher expression in $2.8 \% \mathrm{O}_{2}$ and fully aerobic conditions compared to lower oxygen levels, whereas HXT10 had higher expression at $1 \%$ or more $\mathrm{O}_{2}$. The signals from HXT6, HXT13 and combined HXT15 and HXT16 (HXT15/16) were higher in at least two out of three hypoxic conditions than in either aerobic or anaerobic conditions. The highest transcript levels of HXT6 and HXT3 were seen in $2.8 \% \mathrm{O}_{2}$, whereas the expression of HXT15/16 was reduced at this level of oxygen provision. HXT13 had similar high expression in 1 and $2.8 \% \mathrm{O}_{2}$. None of the hexose transporter genes were upregulated in anaerobic compared to aerobic conditions. The signal for the gene encoding the Snf3p sensor was higher in aerobic compared to anaerobic conditions, and highest in $2.8 \%$
$\mathrm{O}_{2}$, whereas the signal of the RGT2 transcripts was lowest under the hypoxic conditions of 0.5 and $1 \% \mathrm{O}_{2}$.

\section{Expression of transporter encoding genes after a change between aerobic and anaerobic conditions}

The expression of hexose transporter and glucose sensor genes following a change in oxygen provision was studied by switching oxygen provision off or on, in aerobic and anaerobic cultivations, respectively. Following the change from aerobic to anaerobic conditions, samples were taken until a new steady state was achieved (Figure 2A). After the switch from anaerobic to aerobic conditions, the cultivations started to oscillate after 25 hours and the steady state could not be reached. The results are thus reported only for the first 25 hours (Figure 2B).

Clear changes as a response to the removal of oxygen from respiratory cells were seen in the transcript levels of HXT2 to $H X T 7$, although the time scales of the responses varied (Figures 2A and 3). Two of these genes (HXT3 and HXT6) were upregulated and four (HXT2, HXT4, HXT5 and HXT7) downregulated either transiently or permanently as a response to lack of oxygen. The transcript levels of HXT3, HXT4, HXT6 and HXT7 had changed already during the first 10 minutes, while a response in HXT2 expression was seen only after $10 \mathrm{~min}$ but within one hour. Changes observed in HXT5 expression were the slowest to occur; a significant response was seen after 6 hours.

When oxygen was introduced to the anaerobic cultivations (Figures 2B and 3), two of the genes encoding major hexose transporters (HXT3 and HXT6) were downregulated and two (HXT2 and HXT4) were upregulated. HXT5 was upregulated only after a transient downregulation during the first 4 hours after oxygen was provided. HXT7 was slightly upregulated after initial (10 min after oxygen was provided) downregulation. Expression of HXT3, HXT4, HXT6 and HXT7 exhibited rapid responses to a change in environmental oxygen, i.e within $10 \mathrm{~min}$, the earliest sample taken after the change.

The responses of the transporter genes HXT8 to HXT17 were weaker than those of the major hexose transporters (Figures 2A, 2B and 3). In general, these genes were upregulated when oxygen was introduced to anaerobic cultivations, and either downregulated or did not respond when oxygen was removed from aerobic cultures. The expression of HXT15/16, increased only transiently when the culture was adapting to aerobic conditions and permanently following the shift to anaerobic conditions. Of the sensor genes, expression of SNF3 was not affected by change in the oxygen concentration, but RGT2 was downregulated when the cultures experienced lack of oxygen. 

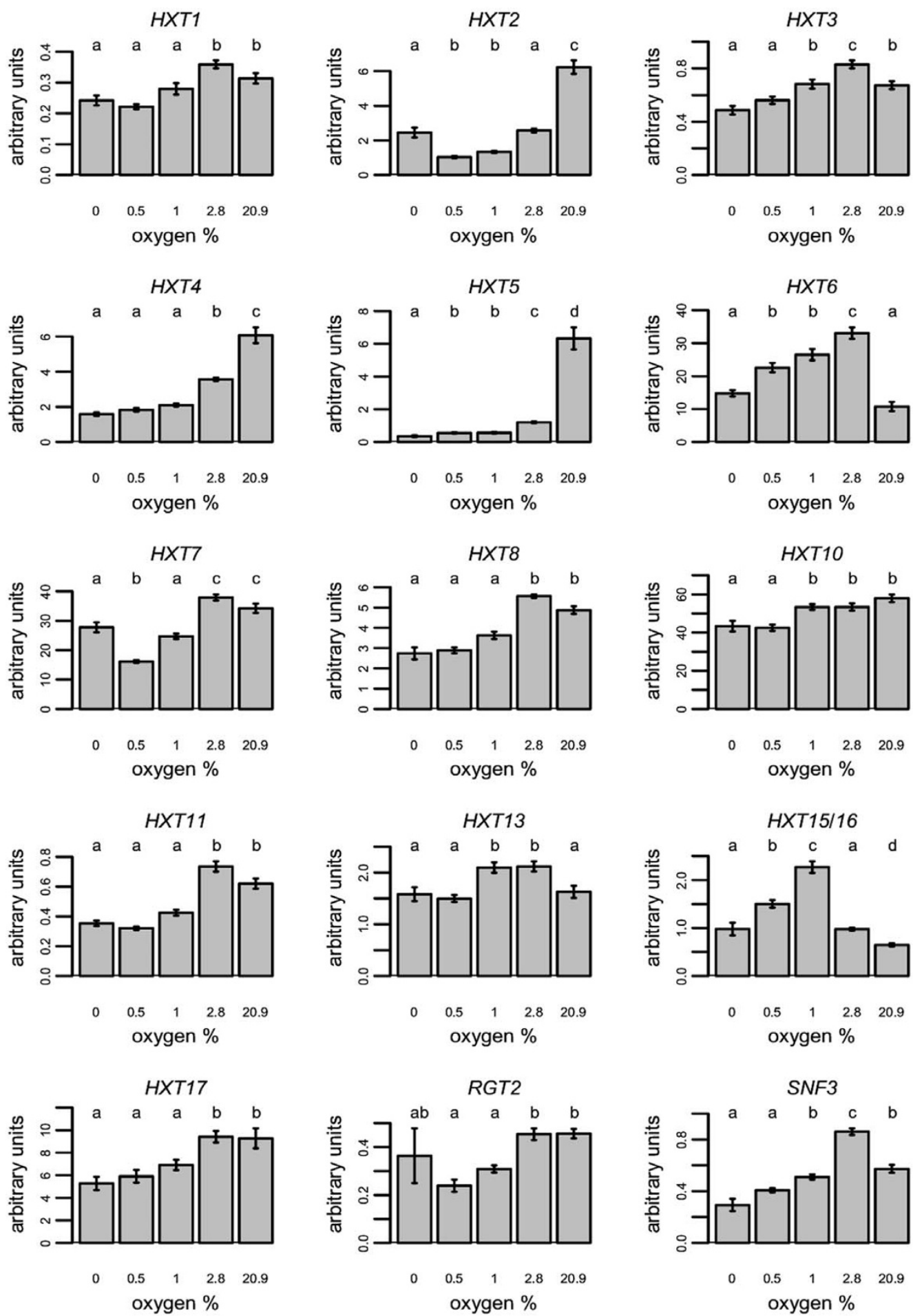

Figure I

The expression of HXT genes at different steady state oxygen levels. The expression of genes encoding hexose transporters and glucose sensors in steady state oxygen concentrations of $0,0.5,1.0,2.8$ or $20.9 \% \mathrm{O}_{2}$ in glucose-limited chemostats $\left(\mathrm{D}=0.10 \mathrm{~h}^{-1}, \mathrm{pH} 5.0,30^{\circ} \mathrm{C}\right.$, and $\mathrm{I} .5 \mathrm{vvm}$ gas flow). Error bars indicate \pm sem for 4 to 8 samples taken during steady states in 2 to 4 cultivations. Values with the same letter (a to e) for the same gene did not differ significantly ( $>>0.05$, Dunnett's T3 multiple range test) from data points showing the same letter. 


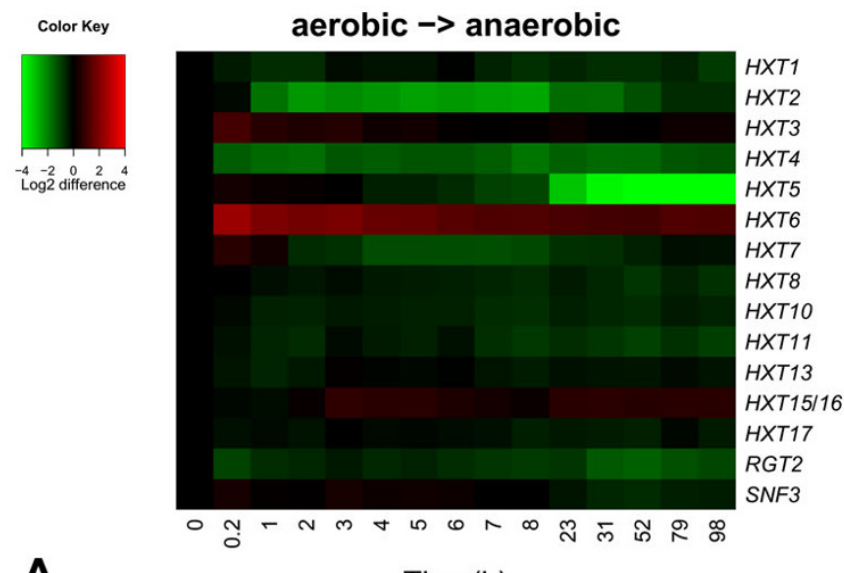

A.

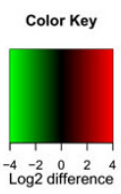

C.

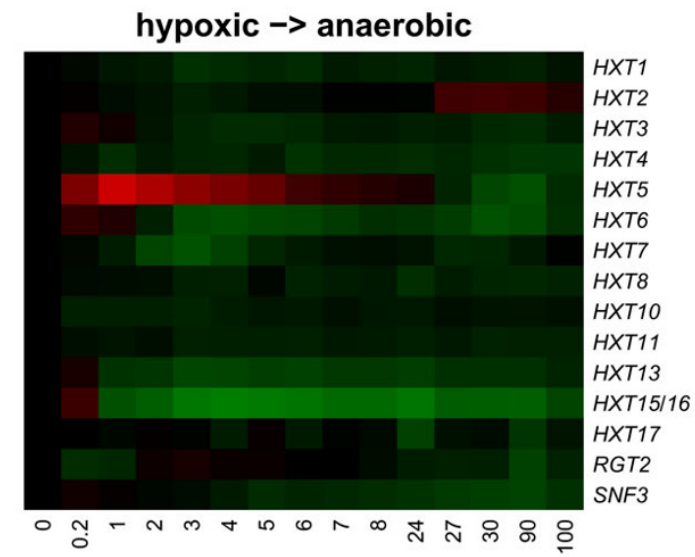

Time(h)

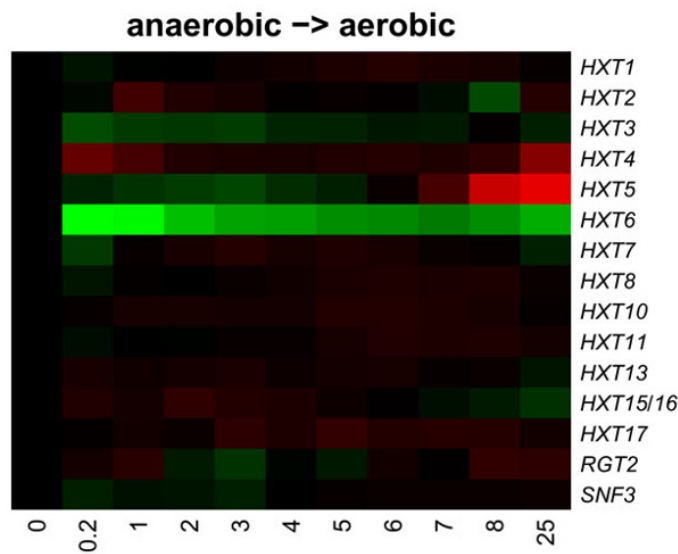

B.

Time(h)

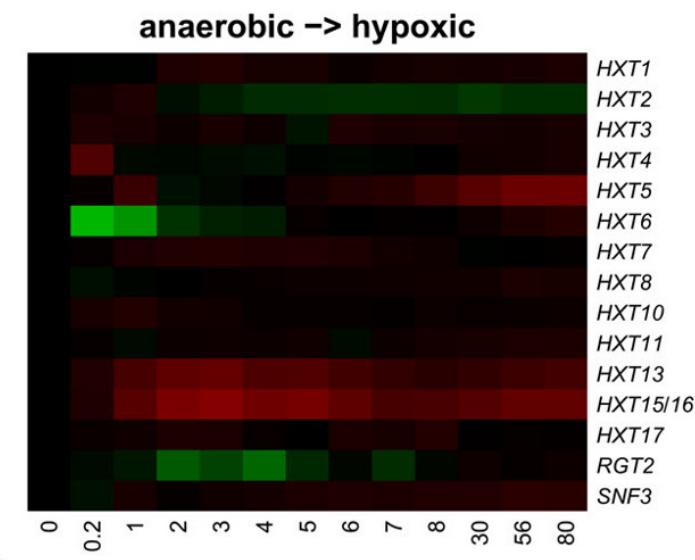

D.

Time(h)

Figure 2

Relative expression levels of $H X T$ genes after a change in oxygen provision. The heatmap of the relative expression levels of genes encoding hexose transporters and glucose sensors after change in oxygen provision. A. Shift from aerobic to anaerobic conditions. B. Shift from anaerobic to aerobic conditions. C. Shift from hypoxic $\left(I \% \mathrm{O}_{2}\right)$ to anaerobic conditions. D. Shift from anaerobic to hypoxic $\left(1 \% \mathrm{O}_{2}\right)$ conditions.

\section{Expression of transporter encoding genes after a change between hypoxic and anaerobic conditions}

The response in expression of HXT and glucose sensor genes was also assessed following a shift from hypoxic $\left(1 \% \mathrm{O}_{2}\right)$ to anaerobic and from anaerobic to hypoxic conditions. Samples were taken until a new steady state was achieved.

The response in the expression of the hexose transporter encoding genes to oxygen depletion was different when the starting point was hypoxic and not fully aerobic (Figures $2 \mathrm{C}-\mathrm{D}$ and 3 ). When oxygen was replaced with nitrogen in the cultures which had been hypoxic, most of the HXT genes were downregulated (Figure 2C). The expression of HXT3, HXT4, HXT6, HXT13 and HXT15/16 was transiently upregulated in cells after $10 \mathrm{~min}$ in anaerobic conditions, but downregulated already after $60 \mathrm{~min}$. Only HXT5 was transiently upregulated for a longer period (the first 7 hours). HXT2 was upregulated towards the end of the adaptation to the anaerobic conditions, around 30 hours after oxygen was depleted.

When hypoxic conditions were introduced to anaerobic cultivations (Figure 2D) the immediate $(10 \mathrm{~min})$ responses were similar to those observed when fully aerobic conditions were introduced (Figure 2B), but the subsequent responses differed. Only the expression of HXT2, HXT5, HXT13 and HXT15/16 changed significantly as the cells approached the hypoxic steady state. HXT5, HXT13 and HXT15/16 were upregulated whereas HXT2 was downregulated. HXT6 and RGT2 were transiently down- 
HXT2

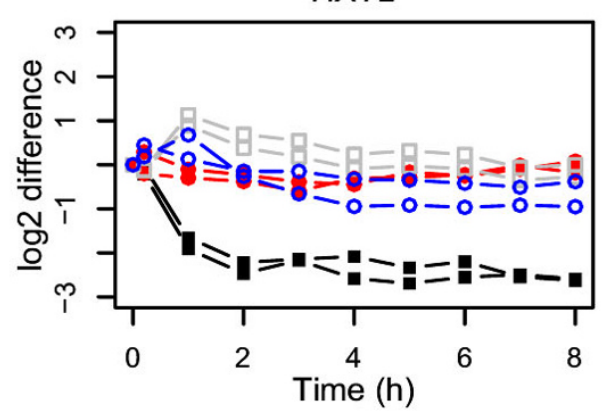

HXT4

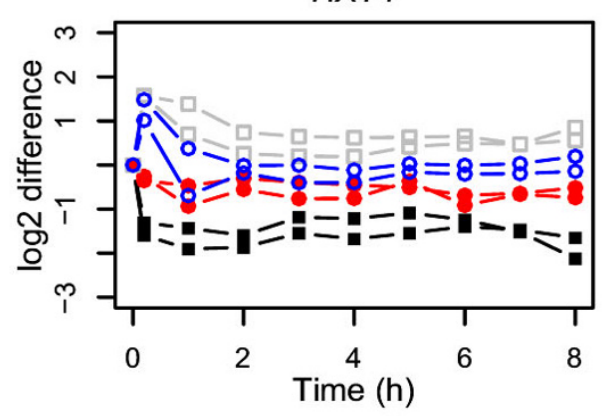

HXT6

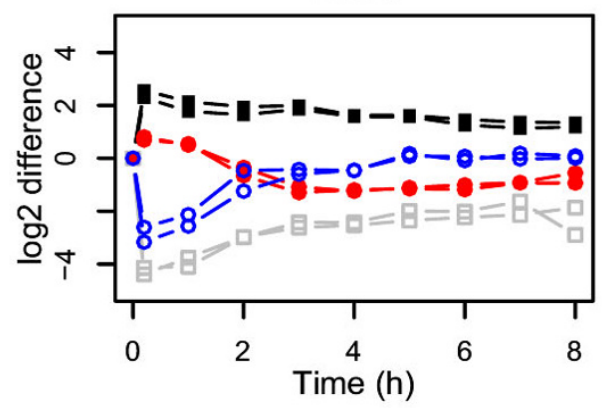

HXT15/16

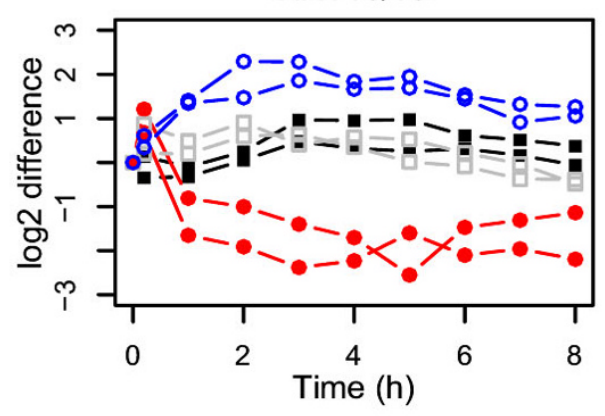

HXT3

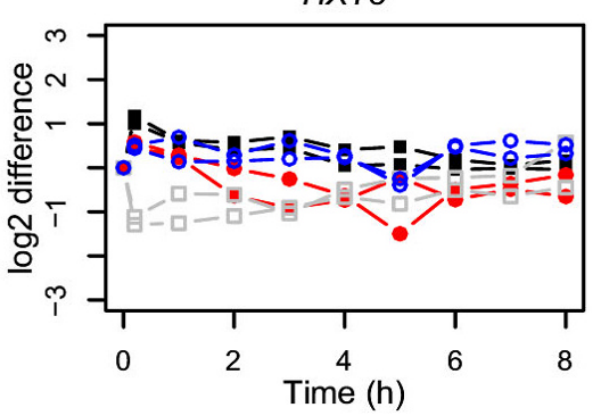

HXT5

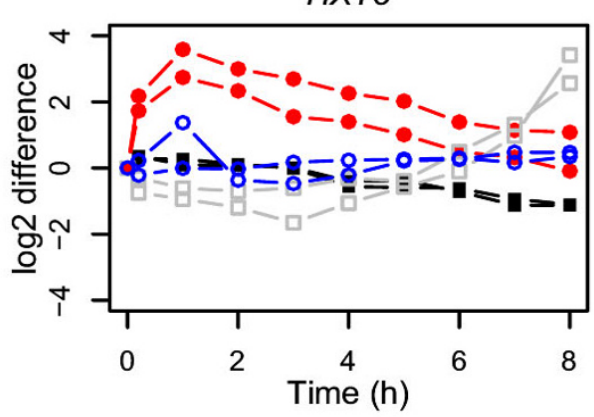

HXT13

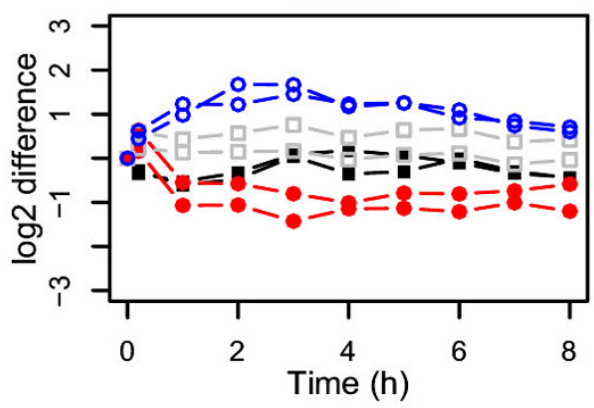

Figure 3

Initial responses of specific $H X T$ genes to the change in oxygen concentration. The relative expression of $H X T 2-$ HXT6, HXTI3 and HXTI5//6 during the first eight hours after the switch in oxygen concentration. Symbols with the same colours represent replicate cultivations carried out in the same conditions. 
regulated during the first 2 and 2-5 hours, respectively, following the switch to hypoxic conditions.

\section{Promoter analysis of the HXT genes induced in hypoxic conditions}

The promoter regions (950 bp upstream from the start codon) of the genes encoding Hxt3p, Hxt6p, Hxt13p, and Hxt15/16p transporters were sequenced in the CEN.PK113-1A strain. The upstream sequences of HXT3, HXT6 and HXT15/16 were identical to those of strain S288C, whose genomic sequence is found in the data banks. In the promoter region of HXT13 there were five separate nucleotides in CEN.PK113-1A that differed from the published sequence of strain S288C. The DNA Pattern [42] web-based tool was used to search the promoters of HXT3, HXT6, HXT13 and HXT15/16 for binding sites of transcription factors known to be involved in hypoxic control. These were LORE (low oxygen response element); 5' ACTCAACAA 3' [43] and HRE (hypoxic response element); 5' BRCGTGVBBB 3' [44]. Two LORE sequences were found in the HXT3 promoter and one in the HXT15/ 16 promoter (one mismatch allowed), whereas one HRE site was found in the HXT13 promoter of both S288C and CEN.PK113-1A strains.

\section{Discussion}

Most of the published data indicate that the expression of the hexose transporter encoding genes of $S$. cerevisiae is mainly regulated by extracellular glucose concentration. In the present paper, the expression of these genes was studied under various oxygen concentrations in glucoselimited chemostats with an extracellular glucose concentration below detection limit and thus not affecting the transcription of HXT genes [20]. When oxygen becomes limiting in a glucose-limited chemostat cultivation, the glucose uptake rate per cell increases. We addressed the question of how the expression level of hexose transporter encoding genes is modified as a result of increased specific glucose consumption rate under oxygen restricted conditions.

In aerobic glucose-limited chemostat cultures grown at a dilution rate of $0.1 \mathrm{~h}^{-1}$, both high and moderately low affinity glucose uptake is detected [20]. A similar result was observed here; expression of the moderately low affinity transporter genes HXT2, HXT4 and HXT5 and of the high affinity transporter genes HXT6 and HXT7 was seen. Expression of HXT1 and HXT3, encoding low affinity transporters was also detected, but on a very low level. In their chemostat study, Diderich and co-workers [20] only detected the expression of HXT2, HXT5 and HXT7. This is most probably due to differences in the sensitivity of the techniques used. We were also able to detect the expression of all other hexose transporter genes except
HXT9, HXT14 and GAL2, although some were only expressed at a very low level.

In anaerobic conditions, the expression of HXT2, HXT4 and HXT5, encoding moderately low affinity transporters was significantly reduced compared to aerobic conditions (Figure 1). This was also seen during transition from aerobic or hypoxic growth to anaerobic growth and agrees with earlier studies which detected only high affinity transport activity in anaerobic glucose-limited chemostat cultivations [20]. In addition to the decreased expression levels of the moderately low affinity transporter encoding genes, the activity of Hxt2p may be modulated towards higher affinity, as both high and moderately low affinity components have been observed in a strain expressing only this transporter gene [4].

Interestingly, the expression of none of the hexose transporter encoding genes was increased in anaerobic compared to aerobic conditions. However, the decrease in the moderately low affinity uptake may result in a relative increase in high affinity uptake, which may allow the higher specific glucose consumption rate to occur in anaerobic conditions. In contrast, Trhxt1, encoding a glucose transporter, is induced by anoxia but not by hypoxia in the filamentous fungus Trichoderma reesei [45]. This may represent a difference between facultative anaerobic and strictly aerobic fungi.

Earlier studies have shown that HXT5 is expressed at a higher level in aerobic than in anaerobic conditions $[20,46]$. Our studies show that the transcription of HXT5 was significantly reduced not only in anaerobic, but also in hypoxic, compared to aerobic conditions. In addition, the regulation of HXT5 following a change in oxygen provision differed from that of other HXT genes. When hypoxic condition became anaerobic, there was a long (7 h), transient upregulation of expression of this gene, that was opposite to the final response of downregulation. It is known that the transcription of HXT5 is not regulated by extracellular glucose concentration, but by growth rate, and that the expression is under the control of STRE and HAP elements in its promoter $[17,19]$. The reduction in specific growth rate for up to $15 \mathrm{~h}$ following a shift from hypoxic to anaerobic conditions may have contributed to the transient upregulation of HXT5, but it should be noted that transient upregulation was not observed following the change from aerobic to anaerobic conditions even though the specific growth rate was similarly reduced. HAP2/3/4/5p elements are needed for the induction of transcription of respiratory genes, like the ones encoding the subunits of cytochrome $c$ oxidase (COX4, COX5a, COX6) and enzymes of the tricarboxylic acid cycle (KGD1, CIT1) [47-51]. These elements are also likely to be 
involved in the aerobic induction of HXT5 seen in this study.

The change in specific growth rate after a change in oxygen provision may also have affected the expression of other hexose transporter encoding genes, directly or inderectly. However, responses to increased oxygen provision (20.9 or $1.0 \%$ ) which occurred within less than 2 hours were independent of specific growth rate, which only increased after this time. Further, specific growth rate increased 2 hours after a change to either 20.9 or $1.0 \%$ oxygen, but for those genes whose transcription was affected by the change between 2 and 8 hours after it occurred, during which time the specific growth rate was increased, the response was generally not the same with 20.9 as with $1.0 \%$ oxygen. Thus it is unlikely that the increase in specific growth rate contributed as much as the increase in oxygen availability in regulation of the hexose transport genes. The decrease in specific growth rate which occurred following a change to anaerobic conditions was also unlikely to be as important as the reduction in oxygen availability in affecting transcription of the hexose transport genes, since a similar reduction in specific growth rate was observed regardless of the initial concentration of oxygen provided, but changes in gene transcription were dependent on the initial oxygen provision.

In hypoxic (0.5-2.8\% oxygen) conditions, the expression of four of the HXT genes was higher than in aerobic conditions. Even with $2.8 \%$ oxygen, compared to aerobic conditions, the expression of HXT3, HXT6, HXT13 and HXT15/16 was higher, whereas the expression of HXT2, HXT4 and HXT5 was lower. In our earlier study we observed that the expression levels of $50 \%$ of the 69 genes of central carbon metabolism which were studied were different under $2.8 \%$ oxygen provision compared to full aeration [32], suggesting that the cell is able to recognise the reduced oxygen level, even though the cultures still maintain a high biomass concentration and a high oxygen uptake rate. Reduced transcript levels of HXT2 and HXT5 and increased transcript levels of HXT3 have previously been observed during respiro-fermentative metabolism at high dilution rates in chemostat cultures [20].

The expression of the high affinity transporter encoding gene HXT6 was relatively strong in all the hypoxic oxygen levels studied compared to aerobic or anaerobic conditions. This was seen in both steady state and non-steady state cultures. In contrast, the expression of the other high affinity transporter encoding gene, HXT7 was very similar in aerobic cultivations and in those receiving $2.8 \%$ oxygen and was low in cultures with less or no oxygen, especially in the culture receiving $0.5 \%$ oxygen. This observation agrees with the earlier observation that even though HXT6 and $H X T 7$ are $99 \%$ identical in their coding regions, they are not co-regulated [20] Indeed, their promoter regions are only $51 \%$ identical.

HXT13 and HXT15/16 exhibited their highest relative expression in hypoxic steady states and also showed clear responses to a shift in oxygen concentration. These genes were induced when hypoxia was introduced after anaerobic conditions and repressed when hypoxic cultivations became anaerobic. The transient responses following shifts between fully aerobic and anaerobic conditions were smaller, reflecting the comparable expression level of these two genes under the two conditions. HXT13 and HXT15 have previously been shown to be slightly induced by non-fermentable carbon sources [24]. In the promoter regions of HXT15 and HXT16 there are LORE (low oxygen response element) [43] sequences with one mismatch to the consensus. These elements could be responsible for the induction in the hypoxic conditions, although the site is located $-900 \mathrm{bp}$ from the start codon of the genes. The promoter of HXT13, on the other hand, contains an HRE (hypoxia response element) sequence, which is responsible for the regulation of hypoxic genes in mammalian cells (e.g. GLUT1 glucose transporter in human) [52-55]. It has recently been speculated that this element is also present in the yeast genome [44].

Only small differences in the expression levels of HXT8, HXT10, HXT11 and HXT17 were seen, either between different steady states or as a response to a change in oxygen concentration. In addition to regulation by glucose concentration (excluding HXT11), the expression levels are known to be affected by change in pH (HXT8, HXT9, HXT11 and HXT17), and HXT11 has been indicated to be involved in multidrug resistance $[7,24,56]$.

Most of the HXT genes had different expression in different oxygen concentrations, either during the steady states or following a change between anaerobic and hypoxic or aerobic conditions. Transcription of HXT2, HXT4 and HXT5 was high in aerobic and that of HXT6, HXT13 and HXT15/16 was high in hypoxic conditions. Additionally, the three former transporter encoding genes clearly had lower expression levels in oxygen-restricted conditions. However, questions concerning the significance of the hypoxic induction, and why the moderately low affinity transporters are expressed in glucose-limited chemostats with very low extracellular glucose concentration, remain to be addressed in future studies. It is tempting to speculate, that the hypoxic induction of expression, in particular that of HXT6, encoding a known high affinity transporter, indicates a response to enhance sugar uptake upon oxygen limitation. The fact that the relative transcript levels of this gene are lower in fully anaerobic compared to hypoxic cultures may not represent the situation at the protein level on the plasma membrane. The 
decreased amount of moderately low affinity transporters, achieved by both regulating the expression of these genes and by degradation of the respective protein(s), under conditions of low oxygen may provide additional membrane space for the high affinity transporters to occupy the membrane and thus increase the glucose uptake rate.

\section{Conclusion}

The expression of hexose transporter encoding genes was affected by change in oxygen provision. The expression of genes encoding moderately low affinity transporters was lower in anaerobic than aerobic conditions. As the expression of none of the hexose transporter encoding genes was increased in anaerobic compared to aerobic conditions it seems that the decrease in the moderately low affinity uptake and consequently the relative increase of high affinity uptake may itself allow a higher specific glucose consumption rate to occur in oxygen restricted and anaerobic conditions. Further, the gene encoding the high affinity transporter Hxt6p and the genes encoding Hxt13p and Hxt 15/16p were upregulated in hypoxic conditions, and the expression of moderately low affinity transporters, particularly that of HXT5, was reduced with only $2.8 \%$ oxygen compared to fully aerobic conditions. The regulation of hexose transporter encoding genes is thus different in oxygen restricted conditions compared to fully anaerobic conditions.

\section{Methods}

\section{Yeast strain and culture conditions}

Saccharomyces cerevisiae CEN.PK113-1A (MATa, URA3, HIS3, LEU2, TRP1, MAL2-8c, SUC2) was grown in 0.8 to $1 \mathrm{~L}$ medium in Braun Biotech International (Sartorius) Biostat $^{\circledR}$ CT (2.5 L working volume) bioreactors in the defined minimal medium described by Verduyn et al.

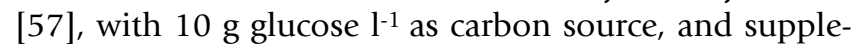
mented with $10 \mathrm{mg}$ ergosterol l-1 and $420 \mathrm{mg}$ Tween $80 \mathrm{l}^{-}$ ${ }^{1}$. BDH silicone antifoam $\left(0.5 \mathrm{~mL} \mathrm{l}^{-1}\right)$ was used to prevent foam production in the cultures. Chemostat cultures were maintained at $\mathrm{D}=0.10 \pm 0.02 \mathrm{~h}^{-1}, \mathrm{pH} 5.0,30^{\circ} \mathrm{C}$, with 1.5 volume gas [volume culture] ${ }^{-1} \mathrm{~min}^{-1}$ (vvm). For cultures which received less than $20.9 \% \mathrm{O}_{2}$ in the gas stream, air was replaced with the equivalent volume of $\mathrm{N}_{2}$, so that total gas flow was maintained constant for all experiments. Cultures which were fed 2.8 or $20.9 \% \mathrm{O}_{2}$ were subject to oscillations. To prevent these, approximately $5 \%$ of the total cell concentration in the bioreactor was added to the culture as cells in mid to late exponential phase at the time when continuous medium feed was started [58]. The cultivations and the culture conditions, biomass determination and metabolite analyses are described in more detail by Wiebe and coworkers [32].

In some cultures, the steady state was disrupted by replacing air $\left(20.9\right.$ or $\left.1.0 \% \mathrm{O}_{2}\right)$ with $100 \% \mathrm{~N}_{2}\left(0 \% \mathrm{O}_{2}\right)$ or by introducing air (20.9 or $1.0 \% \mathrm{O}_{2}$ ) to anaerobic $(100 \%$ $\mathrm{N}_{2}$ ) cultures. When $20.9 \% \mathrm{O}_{2}$ was introduced to an anaerobic culture, dissolved oxygen was present in the culture within less than 15 seconds and was $>70 \%$ within less than 2 minutes. When $1.0 \% \mathrm{O}_{2}$ was introduced to an anaerobic culture, dissolved oxygen was measurable in the culture within less than 2 minutes and for more than $2 \mathrm{~h}$ before returning to 0 . When $20.9 \% \mathrm{O}_{2}$ was replaced by $\mathrm{N}_{2}$, the dissolved oxygen decreased from $\sim 80 \%$ to 0 within less than 2 minutes. No change in dissolved oxygen, which was 0 , occurred when $1 \% \mathrm{O}_{2}$ was replaced by $\mathrm{N}_{2}$.

The dilution rate and other conditions were maintained constant following a shift in conditions, but the differences in biomass yield in $0 \%(0.12 \mathrm{Cmol}$ biomass [Cmol glucose $\left.\left.^{-1}\right), 1.0 \%(0.36 \mathrm{Cmol} \text { biomass [Cmol glucose }]^{-1}\right)$ and $\left.20.9 \%(0.60 \mathrm{Cmol} \text { biomass [Cmol glucose }]^{-1}\right) \mathrm{O}_{2}$ [32] resulted in changes in specific growth rate for up to approximately $15 \mathrm{~h}$ following the shift. In cultures provided with either 20.9 or $1.0 \% \mathrm{O}_{2}$, no increase in specific growth rate was observed during the first $2 \mathrm{~h}$ following the addition of $\mathrm{O}_{2}$. Between 2 and approximately $10 \mathrm{~h}$, cells in cultures receiving $20.9 \% \mathrm{O}_{2}$ grew at $0.32 \mathrm{~h}^{-1}$, while those receiving $1.0 \% \mathrm{O}_{2}$ grew at $0.21 \mathrm{~h}^{-1}$. After $10 \mathrm{~h}$, growth continued at $0.10 \mathrm{~h}^{-1}$. In cultures which were made anaerobic, the specific growth rate decreased to 0.06 $\mathrm{h}^{-1}$ almost immediately after $\mathrm{O}_{2}$ was replaced with $\mathrm{N}_{2}$ and returned to $0.10 \mathrm{~h}^{-1}$ after approximately $15 \mathrm{~h}$ [32]. During these time intervals the cultures also experienced changes in extracellular metabolite concentrations, with ethanol and glycerol concentrations increasing in cultures which became anaerobic [32] and decreasing in cultures which became aerobic or hypoxic. Glycerol consumption did not occur, but ethanol consumption was observed in aerobic cultures. Ethanol production continued in the hypoxic cultures.

Oscillations occurred in cultures which were maintained in steady state anaerobic conditions and then provided with $20.9 \% \mathrm{O}_{2}$ approximately $25 \mathrm{~h}$ after $\mathrm{O}_{2}$ was provided. Fresh, exponentially growing cells were not added to prevent oscillations since the transcript levels in the added cells may have affected the overall results disproportionately.

\section{Transcriptional analysis}

Transcriptional analysis was performed with the TRanscript analysis with aid of Affinity Capture (TRAC) assay described by Rautio et al. [59]. Total mRNA was extracted from $10 \mathrm{~mL}$ cell culture samples $(10-50 \mathrm{mg}$ DW) which had been rapidly frozen in liquid $\mathrm{N}_{2}$ and stored at $-80^{\circ} \mathrm{C}$. GeneScan-120LIZ size standard (Applied Biosystems, USA) was added to each sample to calibrate the separation of the detection probes by size. In addition, in vitro synthe- 
sized mRNA (MEGAscript transcription kit; Ambion, USA) of the Escherichia coli traT gene was added to each sample $\left(1.5 \mathrm{fmol}[100 \mu \mathrm{l}]^{-1}\right)$ so that the results for each probe in any analysis could be correlated to this internal standard, eliminating experimental variation in different hybridizations and samples. Probes were divided into 2 probe pools with 8 and 9 probes per pool. The identity of the probes was determined by the migration behaviour and the quantity by the peak area. Total polyA RNA was quantified from the cell lysates, after eluting the polyA RNA in dimethyl pyrocarbonate (DMPC) treated $\mathrm{H}_{2} \mathrm{O}$, using the RiboGreen RNA quantification kit (Molecular Probes, the Netherlands). Individual mRNA expression levels are given as the standardised (using traT internal standard) amount per total polyA RNA.

\section{Probes for TRAC analysis}

The probes used in the TRAC analyses are listed in Table 1. They were designed using the mathematical algorithms presented in Kivoja et al. [60]. The probes of HXT1-5, HXT8-10, GAL2, RGT2 and SNF3 were designed to hybridise to the 3 ' end of the coding regions. The coding regions of HXT13 and HXT17 as well as HXT9 and HXT11 have sequence similarity of $97 \%$. Only the 5 ' ends of the coding region of these genes differ enough to enable the design of specific probes. HXT6 and HXT7 are 99\% identical within the coding region, but differences can be found in the $3^{\prime}$ flank of the genes. The flanking region of HXT7 is AT rich which leads to a melting temperature of the probe $\left(50^{\circ} \mathrm{C}\right)$ that is lower than that of the other probes $\left(62-71^{\circ} \mathrm{C}\right)$. HXT15 and HXT16 are identical within their coding regions and within $1 \mathrm{~kb}$ in both directions from the cod- ing region, except one nucleotide. Therefore only one probe was designed which detected both of these genes.

\section{Sequencing of promoter regions}

The following oligonucleotides were used in PCR of promoter fragments from the genomic DNA of CEN.PK1131A: HXT3 promoter forward1 5'ACCGGTATATCAAATGGCGGTGTA 3', HXT3 promoter reverse 5'TCAGGCATGTTCATTACCTGAGAG 3', HXT6 promoter forward1 5'TGGCATCAAATTTGGGAA 3', HXT6 promoter reverse 5'GAGAGATGCTCCACAGGA 3', HXT13 promoter forward 1 5'TGCTGCAATTTGCTATTT 3', HXT13 promoter reverse 5'CATCTCCATCGCTATCAA 3', HXT15 promoter forward 1 5'CCATTTTTTCAGAATCCT 3', HXT15 promoter reverse 5'CTGTTTAGATTATCTGCA 3'. Three parallel PCRreactions for each of the $H X T$ promoters sequenced were carried out using Dynazyme Ext or Phusion high fidelity polymerases (Finnzymes, Finland), and purified PCRfragments were sequenced using the same oligonucleotides that were used in the PCR-reactions. In addition, the following oligonucleotides were used in the sequencing: HXT3 promoter forward2 5'GGAACATTCTAGCTCGTT 3', HXT6 promoter forward2 5'TACTTGGAAATTAATGTA 3', HXT13 promoter forward2 5'ATCATTTGTCGTGTTCCT $3{ }^{\prime}$ and HXT15 promoter forward2 5'CCAAATATCTTATACGTT 3'.

\section{Data analysis and graphs}

Statistical analysis of the data was carried out using SPSS software (version 14.0, SPSS Inc, USA,). Analysis of variance (ANOVA) and Dunnett's T3 multiple comparison test were used for the comparative analysis of the data. All graphs were prepared using the R environment $[61,62]$.

Table I: Probes used in the TRAC analysis

\begin{tabular}{|c|c|c|}
\hline Gene & Probe sequence & Position $^{\mathrm{a}}$ \\
\hline HXTI & ATGGTCAGGTGGGCATTTGTTAACTTTAGCTAA & 826 \\
\hline HXT2 & TAACTCACCCCAAGAAGCGTTACCG & 891 \\
\hline HXT3 & ATACCAATGGCACCATATAACAAACAGTTACGACGTC & 1145 \\
\hline HXT4 & CTGGATCGTCTGCGCTGACCTTATTTGAAAGAGCAATAG & 842 \\
\hline HXT5 & ATCATACCCATTAGTGTACGTCTGAAC & 1026 \\
\hline HXT6 & CATCTTGCCATACAATATAAATCGTAAGGGTTCAT & +78 \\
\hline HXT7 & GTATATATTAAAAACGTATTTACTTTTCAAGATATCATTAAAA & +83 \\
\hline НХT8 & AGAAGGGTTTCTCGTCATGCTGTAATTTTTCGT & $|66|$ \\
\hline HXT9 & ATGCTCAGTTTTTACAGATGGTGCATTTGCTACTGAG & 60 \\
\hline HXTIO & TGGCCAAAGACCTTCTAGCTTCTTCATACTTACCTTTTTCTAC & 742 \\
\hline HXTII & GGCTCATTGGCATCTAGGTTAAGGGAATT & 109 \\
\hline HXTI3 & ATCTCGAACATCTCCATCGCTATCAATAGAGGATT & 14 \\
\hline HXTI4 & TATGGCCCTCGTTCTTAGAGGGAACAATTCG & 1386 \\
\hline HXT/5//6 & GCCCATGTCGTTGCAAAGCAGAATATATAGAAGCATGTG & 1284 \\
\hline HXTI7 & GACCATCCTGAATATCTCTATCACT & 19 \\
\hline SNF3 & TCTGTACTAGTAGGAATATCAACACGTTCTG & 1892 \\
\hline RGT2 & TTCACTTGTTTTGAAACAATCTAAAAGTGTTGACGGGCCGA & 1007 \\
\hline GAL2 & AGAGGCCAACGCCATACATTTCGACTTGA & 1397 \\
\hline
\end{tabular}

a The start position of the probe inside the coding region. + indicates that the probe sequence is on the 3 ' side of the stop codon and the position is given relative to the stop codon. 


\section{Authors' contributions}

ER, LR and MP conceived the study. ER carried out the transcriptional, promoter and statistical analyses, participated in the fermentations and drafted the manuscript. AT and MGW carried out the fermentations and MGW revised the manuscript. LR supervised the work and revised the manuscript. All authors read and approved the final manuscript.

\section{Acknowledgements}

We thank Pirjo Tähtinen, Outi Könönen, Eila Leino and Tarja Hakkarainen for excellent technical assistance. The financial support of Tekes, Finnish Funding Agency for Technology and Innovation (Project numbers 40I 35/04 and 40537/05) and Academy of Finland, (Centre of Excellence, Industrial Biotechnology 2000-2005; project number 214568, and SYSBIO programme; project number 207435) is gratefully acknowledged.

\section{References}

I. Kruckeberg AL: The hexose transporter family of Saccharomyces cerevisiae. Arch Microbiol 1996, 166:283-292.

2. Özcan S, Dover J, Rosenwald AG, Wolfl S, Johnston M: Two glucose transporters in Saccharomyces cerevisiae are glucose sensors that generate a signal for induction of gene expression. Proc Natl Acad Sci USA 1996, 93: I2428-I 2432.

3. Reifenberger E, Freidel K, Ciriacy M: Identification of novel HXT genes in Saccharomyces cerevisiae reveals the impact of individual hexose transporters on glycolytic flux. Mol Microbiol 1995, 16:157-167.

4. Reifenberger E, Boles E, Ciriacy M: Kinetic characterization of individual hexose transporters of Saccharomyces cerevisiae and their relation to the triggering mechanisms of glucose repression. Eur / Biochem 1997, 245:324-333.

5. Platt A, Reece RJ: The yeast galactose genetic switch is mediated by the formation of a Gal4p-Gal80p-Gal3p complex. EMBO J 1998, 17:4086-4091.

6. Wieczorke R, Krampe S, Weierstall T, Freidel K, Hollenberg CP, Boles E: Concurrent knock-out of at least 20 transporter genes is required to block uptake of hexoses in Saccharomyces cerevisiae. FEBS Lett 1999, 464:123-128.

7. Özcan S, Johnston M: Function and regulation of yeast hexose transporters. Microbiol Mol Biol Rev 1999, 63:554-569.

8. Özcan S, Johnston M: Three different regulatory mechanisms enable yeast hexose transporter $(H X T)$ genes to be induced by different levels of glucose. Mol Cell Biol 1995, 1 5: I564-1572.

9. Lakshmanan J, Mosley AL, Özcan S: Repression of transcription by RgtI in the absence of glucose requires Std I and Mth I. Curr Genet 2003, 44:19-25.

10. Polish JA, Kim JH, Johnston M: How the Rgt I transcription factor of Saccharomyces cerevisiae is regulated by glucose. Genetics 2005, 169:583-594.

II. Schmidt MC, McCartney RR, Zhang X, Tillman TS, Solimeo H, Wolfl $S$, Almonte C, Watkins SC: Std I and Mth I proteins interact with the glucose sensors to control glucose-regulated gene expression in Saccharomyces cerevisiae. Mol Cell Biol 1999, 19:456I-457|.

12. $\mathrm{Kim} \mathrm{JH}$, Brachet $\mathrm{V}$, Moriya $\mathrm{H}$, Johnston M: Integration of transcriptional and posttranslational regulation in a glucose signal transduction pathway in Saccharomyces cerevisiae. Eukaryot Cell 2006, 5: 167-173.

13. $\mathrm{Kim} \mathrm{JH}$, Johnston $\mathrm{M}$ : Two glucose-sensing pathways converge on Rgt I to regulate expression of glucose transporter genes in Saccharomyces cerevisiae. J Biol Chem 2006, 28 I:26|44-26I49.

14. Palomino A, Herrero P, Moreno F: Tpk3 and SnfI protein kinases regulate Rgtl association with Saccharomyces cerevisiae HXK2 promoter. Nucleic Acids Res 2006, 34:| 427-I438.

15. Özcan S, Leong T, Johnston M: Rgt Ip of Saccharomyces cerevisiae, a key regulator of glucose-induced genes, is both an activator and a repressor of transcription. Mol Cell Biol 1996, 16:6419-6426.
16. Kaniak A, Xue Z, Macool D, Kim JH, Johnston M: Regulatory network connecting two glucose signal transduction pathways in Saccharomyces cerevisiae. Eukaryot Cell 2004, 3:22I-23I.

17. Verwaal R, Arako M, Kapur R, Verkleij AJ, Verrips CT, Boonstra J: HXT5 expression is under control of STRE and HAP elements in the HXT5 promoter. Yeast 2004, $21: 747-757$.

18. Verwaal R, Paalman JW, Hogenkamp A, Verkleij AJ, Verrips CT, Boonstra J: HXT5 expression is determined by growth rates in Saccharomyces cerevisiae. Yeast 2002, 19:1029-1038.

19. Diderich JA, Schuurmans JM, Van Gaalen MC, Kruckeberg AL, Van Dam K: Functional analysis of the hexose transporter homologue HXT5 in Saccharomyces cerevisiae. Yeast 200I, 18:1515-1524.

20. Diderich JA, Schepper M, van Hoek P, Luttik MA, van Dijken JP, Pronk JT, Klaassen P, Boelens HF, de Mattos MJ, van Dam K, Kruckeberg AL: Glucose uptake kinetics and transcription of HXT genes in chemostat cultures of Saccharomyces cerevisiae. I Biol Chem 1999, 274:I5350-15359.

21. Nourani A, Wesolowski-Louvel M, Delaveau T, Jacq C, Delahodde A: Multiple-drug-resistance phenomenon in the yeast Saccharomyces cerevisiae: involvement of two hexose transporters. Mol Cell Biol 1997, I7:5453-5460.

22. Gross C, Kelleher M, lyer VR, Brown PO, Winge DR: Identification of the copper regulon in Saccharomyces cerevisiae by DNA microarrays. J Biol Chem 2000, 275:32310-32316.

23. Jungmann J, Reins HA, Lee J, Romeo A, Hassett R, Kosman D, Jentsch $\mathrm{S}: \mathrm{MACI}$, a nuclear regulatory protein related to $\mathrm{Cu}$-dependent transcription factors is involved in $\mathrm{Cu} / \mathrm{Fe}$ utilization and stress resistance in yeast. EMBO J I 993, I 2:505 I-5056.

24. Greatrix BW, van Vuuren HJ: Expression of the HXTI3, HXTI5 and HXTI7 genes in Saccharomyces cerevisiae and stabilization of the HXTI gene transcript by sugar-induced osmotic stress. Curr Genet 2006, 49:205-2 17.

25. Liu Z, Boles E, Rosen BP: Arsenic trioxide uptake by hexose permeases in Saccharomyces cerevisiae. J Biol Chem 2004, 279: $|73| 2-173 \mid 8$.

26. Kruckeberg AL, Ye L, Berden JA, van Dam K: Functional expression, quantification and cellular localization of the Hxt2 hexose transporter of Saccharomyces cerevisiae tagged with the green fluorescent protein. Biochem J 1999, 339(Pt 2):299-307.

27. Krampe $S$, Boles $E$ : Starvation-induced degradation of yeast hexose transporter Hxt7p is dependent on endocytosis, autophagy and the terminal sequences of the permease. FEBS Lett 2002, 5 1 3:193-196.

28. Ye L, Berden JA, van Dam K, Kruckeberg AL: Expression and activity of the Hxt7 high-affinity hexose transporter of Saccharomyces cerevisiae. Yeast 200I, I8:I257-1267.

29. Krampe S, Stamm O, Hollenberg CP, Boles E: Catabolite inactivation of the high-affinity hexose transporters Hxt6 and Hxt7 of Saccharomyces cerevisiae occurs in the vacuole after internalization by endocytosis. FEBS Lett 1998, 44 I:343-347.

30. van Suylekom D, van Donselaar E, Blanchetot C, Nguyen Do Ngoc L, Humbel BM, Boonstra J: Degradation of the hexose transporter Hxt5p in Saccharomyces cerevisiae. Biol Cell 2007, 99:13-23.

31. Rogers PJ, Stewart PR: Energetic efficiency and maintenance. Energy characteristics of Saccharomyces cerevisiae (wild type and petite) and Candida parapsilosis grown aerobically and micro-aerobically in continuous culture. Arch Microbiol 1974, 99:25-46

32. Wiebe MG, Rintala E, Tamminen A, Simolin H, Salusjärvi L, Toivari M, Kokkonen JT, Kiuru J, Ketola RA, Jouhten P. Huuskonen A, Maaheimo H, Ruohonen L, Penttilä M: Central carbon metabolism of Saccharomyces cerevisiae in anaerobic, oxygen-limited and fully aerobic steady-state conditions and following a shift to anaerobic conditions. FEMS Yeast Res 2008, 8(I): $140-54$.

33. Davies SE, Brindle KM: Effects of overexpression of phosphofructokinase on glycolysis in the yeast Saccharomyces cerevisiae. Biochemistry 1992, 3 1:4729-4735

34. Ernandes JR, De Meirsman C, Rolland F, Winderickx J, de Winde J, Brandao RL, Thevelein JM: During the initiation of fermentation overexpression of hexokinase PII in yeast transiently causes a similar deregulation of glycolysis as deletion of TpsI. Yeast 1998, 14:255-269.

35. Blazquez MA, Lagunas R, Gancedo C, Gancedo JM: Trehalose-6phosphate, a new regulator of yeast glycolysis that inhibits hexokinases. FEBS Lett 1993, 329:5 I-54. 
36. Schaaff I, Heinisch J, Zimmermann FK: Overproduction of glycolytic enzymes in yeast. Yeast 1989, 5:285-290.

37. Fell DA, Thomas S: Physiological control of metabolic flux: the requirement for multisite modulation. Biochem J I995, 3 I I (Pt I):35-39.

38. Reijenga KA, Snoep JL, Diderich JA, van Verseveld HW, Westerhoff HV, Teusink B: Control of glycolytic dynamics by hexose transport in Saccharomyces cerevisiae. Biophys J 200I, 80:626-634.

39. Diderich JA, Teusink B, Valkier J, Anjos J, Spencer-Martins I, van Dam K, Walsh MC: Strategies to determine the extent of control exerted by glucose transport on glycolytic flux in the yeast Saccharomyces bayanus. Microbiology 1999, I45(Pt 1 2):3447-3454.

40. Ye L, Kruckeberg AL, Berden JA, van Dam K: Growth and glucose repression are controlled by glucose transport in Saccharomyces cerevisiae cells containing only one glucose transporter. J Bacteriol 1999, I 81:4673-4675.

4I. Elbing K, Larsson C, Bill RM, Albers E, Snoep JL, Boles E, Hohmann S, Gustafsson L: Role of hexose transport in control of glycolytic flux in Saccharomyces cerevisiae. Appl Environ Microbiol 2004, 70:5323-5330.

42. van Helden J, Andre B, Collado-Vides J: Extracting regulatory sites from the upstream region of yeast genes by computational analysis of oligonucleotide frequencies. J Mol Biol 1998, 28I:827-842.

43. Vasconcelles MJ, Jiang Y, McDaid K, Gilooly L, Wretzel S, Porter DL Martin CE, Goldberg MA: Identification and characterization of a low oxygen response element involved in the hypoxic induction of a family of Saccharomyces cerevisiae genes. Implications for the conservation of oxygen sensing in eukaryotes. J Biol Chem 200I, 276: | 4374- | 4384.

44. Ferreira TC, Hertzberg L, Gassmann M, Campos EG: The yeast genome may harbor hypoxia response elements (HRE). Comp Biochem Physiol C Toxicol Pharmacol 2007, 146:255-263.

45. Ramos AS, Chambergo FS, Bonaccorsi ED, Ferreira AJ, Cella N, Gombert AK, Tonso A, El-Dorry $\mathrm{H}$ : Oxygen- and glucosedependent expression of Trhxtl, a putative glucose transporter gene of Trichoderma reesei. Biochemistry 2006, 45(26):8।84-92.

46. ter Linde JJ, Liang H, Davis RW, Steensma HY, van Dijken JP, Pronk JT: Genome-wide transcriptional analysis of aerobic and anaerobic chemostat cultures of Saccharomyces cerevisiae. Bacteriol 1999, I 8 I:7409-74|3.

47. Forsburg SL, Guarente L: Communication between mitochondria and the nucleus in regulation of cytochrome genes in the yeast Saccharomyces cerevisiae. Annu Rev Cell Biol 1989, 5:153-180

48. Hodge MR, Kim G, Singh K, Cumsky MG: Inverse regulation of the yeast COX5 genes by oxygen and heme. Mol Cell Biol 1989, 9:1958-1964.

49. Repetto B, Tzagoloff A: Structure and regulation of KGDI, the structural gene for yeast alpha-ketoglutarate dehydrogenase. Mol Cell Biol 1989, 9:2695-2705.

50. Rosenkrantz M, Kell CS, Pennell EA, Devenish LJ: The HAP2,3,4 transcriptional activator is required for derepression of the yeast citrate synthase gene, CITI. Mol Microbiol 1994, 13:|19-131.

51. Trawick JD, Wright RM, Poyton RO: Transcription of yeast COX6, the gene for cytochrome c oxidase subunit VI, is dependent on heme and on the HAP2 gene. J Biol Chem 1989, 264:7005-7008.

52. Takagi H, King GL, Aiello LP: Hypoxia upregulates glucose transport activity through an adenosine-mediated increase of GLUTI expression in retinal capillary endothelial cells. Diabetes 1998, 47: | 480-1488.

53. Ouiddir A, Planes C, Fernandes I, VanHesse A, Clerici C: Hypoxia upregulates activity and expression of the glucose transporter GLUTI in alveolar epithelial cells. Am J Respir Cell Mol Biol 1999, 21:710-718.

54. Royer C, Lachuer J, Crouzoulon G, Roux J, Peyronnet J, Mamet Pequignot J, Dalmaz Y: Effects of gestational hypoxia on mRNA levels of Glut3 and Glut4 transporters, hypoxia inducible factor- I and thyroid hormone receptors in developing rat brain. Brain Res 2000, 856: I19-128.

55. Hayashi M, Sakata M, Takeda T, Yamamoto T, Okamoto Y, Sawada K, Kimura A, Minekawa R, Tahara M, Tasaka K, Murata Y: Induction of glucose transporter I expression through hypoxia-inducible factor Ialpha under hypoxic conditions in trophoblastderived cells. J Endocrinol 2004, I 83: | 45- I54.

56. Serrano R, Ruiz A, Bernal D, Chambers JR, Arino J: The transcriptional response to alkaline $\mathrm{pH}$ in Saccharomyces cerevisiae: evidence for calcium-mediated signalling. Mol Microbiol 2002, 46:1319-1333.

57. Verduyn C, Postma E, Scheffers WA, Van Dijken JP: Effect of benzoic acid on metabolic fluxes in yeasts: a continuous-culture study on the regulation of respiration and alcoholic fermentation. Yeast 1992, 8:501-517.

58. Zamamiri AQ, Birol G. Hjortso MA: Multiple stable states and hysteresis in continuous, oscillating cultures of budding yeast. Biotechnol Bioeng 200I, 75:305-3I2.

59. Rautio |l, Kataja K, Satokari R, Penttilä M, Söderlund H, Saloheimo M: Rapid and multiplexed transcript analysis of microbial cultures using capillary electrophoresis-detectable oligonucleotide probe pools. I Microbiol Methods 2006, 65:404-4I6.

60. Kivioja T, Arvas M, Kataja K, Penttilä M, Söderlund H, Ukkonen E: Assigning probes into a small number of pools separable by electrophoresis. Bioinformatics 2002, I 8(SuppI I):S199-206.

61. R Development Core Team: R: A Language and Environment for Statistical Computing. R Foundation for Statistical Computing. In Version 2.3.0 Vienna, Austria; 2005.

62. Warnes GR: gplots: Various R programming tools for plotting data. Included $R$ source code and/or documentation contributed by Ben Bolker and Thomas Lumley. $R$ package version 2.3.0 2006 .

Publish with BioMed Central and every scientist can read your work free of charge

"BioMed Central will be the most significant development for disseminating the results of biomedical research in our lifetime. "

Sir Paul Nurse, Cancer Research UK

Your research papers will be:

- available free of charge to the entire biomedical community

- peer reviewed and published immediately upon acceptance

- cited in PubMed and archived on PubMed Central

- yours - you keep the copyright
BioMedcentral 